\title{
ANALYSIS OF MODULATOR CIRCUITS BASED ON MULTI-DIMENSIONAL FOURIER TRANSFORMATION
}

\author{
Akio USHIDA, Yoshihiro YAMAGAMI, Yoshifumi NISHIO \\ Department of Electrical and Electronic Engineering, \\ Tokushima University, Tokushima, 770 JAPAN
}

\begin{abstract}
There are many communication circuits driven by multitone signals such as modulator and mixer. If the input frequency components are largely different in each other, the brute force numerical method will take an enormous computation time to get the steady-state responses. In this paper, we show a SPICE oriented algorithm based on multi-dimensional Fourier transformation, where all of the circuit analyses such as dc- and ac-analysis in the algorithm are carried out with SPICE. On the other hand, a very simple sensitivity analysis and 2-dimensional FFT are carried out by a Fortran program (or C program). We found that the convergence ratio of our algorithm is sufficiently large, and can be applied to wide class of communication circuits.
\end{abstract}

\section{INTRODUCTION}

Many communication circuits, such as modulators, mixers and frequency converters, are driven by multi-tone signals. There are 2 basic approaches for the computation of the steady-state responses: (1) frequency-domain approach [1-2] and (2) time-domain approach [3-4]. The former can be applied only to weakly nonlinear circuits, because the scale of determining equations becomes very large for strongly nonlinear circuits. The latter is based on numerical integration techniques, that can be efficiently applied to circuits having a few number of the state variables.

Generally, modulators and mixers are driven by two input signals, namely,the high frequency carrier and the low frequency modulating signal. In this case, if we use a brute force method (transient analysis) for getting the steady-state response, it will take an enormous computational time, because the step size must be chosen sufficiently small depending on the carrier signal. Consider an example such that a ratio of the two input frequencies is $f_{2} / f_{1}=1000$. If the step size is chosen $h=T_{2} / 100$ for $T_{2}=1 / f_{2}$, it will take $1000 \times 100$ numerical integrations for only one period $\left(T_{1}=1 / f_{1}\right)$.
A new SPICE oriented method is presented in this paper which is based on both 2-dimensional Fourier transformation and frequency-domain relaxation methods. Assume that a given circuit is composed of nonlinear resistive sub-networks and reactive elements such as capacitors and inductors. At first, using the substitution sources, the circuit is partitioned into two groups, namely, the nonlinear resistive subnetworks and the reactive elements. The substitution waveforms are described by 2-dimensional Fourier expansions, and the coefficients are calculated by the relaxation method.

We have developed a very simple simulator consisting of SPICE and a Fortran program, where all of the circuit analysis are implemented by SPICE. On the other hand, very simple sensitivity analysis and 2-dimensional FFT are carried out by the Fortran program.

\section{BASIC APPROACH}

To focus on the main idea of our relaxation method, consider a circuit as shown in Fig.1(a) ${ }^{1}$. Now, assume the two inputs $e(t)$ and $j(t)$ contain two independent frequency components $\omega_{1}$ and $\omega_{2}$.

Then, the substitution sources at the partitioning point will be generally assumed of the form

$$
\begin{gathered}
v_{C}(t)=V_{C, 0}+\sum_{k=1}^{M}\left\{V_{C, 2 k-1} \cos \nu_{k} t+V_{C, 2 k} \sin \nu_{k} t\right\} \\
i_{L}(t)=I_{L, 0}+\sum_{k=1}^{M}\left\{I_{L, 2 k-1} \cos \nu_{k} t+I_{L, 2 k} \sin \nu_{k} t\right\} \\
\nu \equiv m_{1 k} \omega_{1}+m_{2 k} \omega_{2}
\end{gathered}
$$

${ }^{1}$ Generally, integrated circuits are composed of capacitors and resistive elements such as transistors and diodes. If in this case, it contains large capacitances, the transient response will continue for a large period, and it take long computation time to get the steady-state by the brute force method. Therefore, we partitioned the circuit into two groups containing nonlinear resistive circuit and reactive elements respectively, as shown in Fig.1(b) 


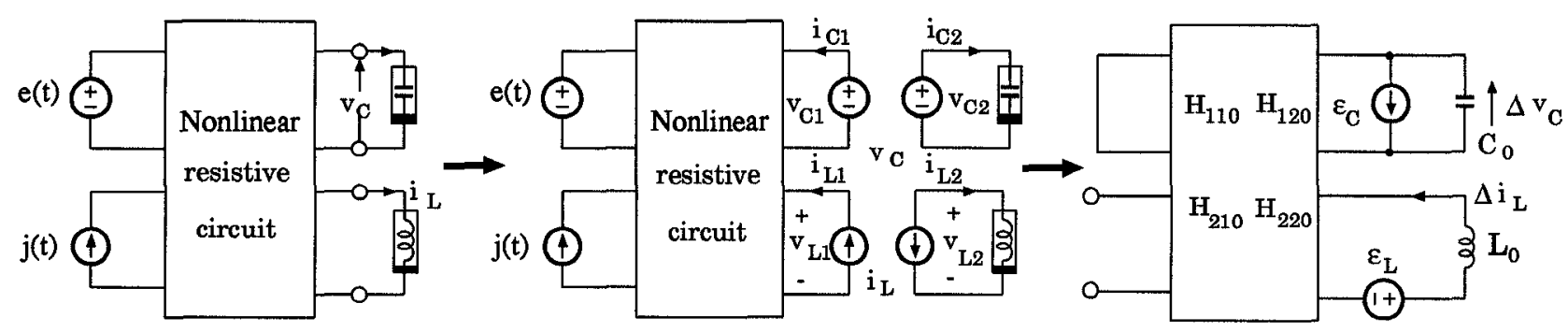

(a) Nonlinear circuit

(b) Circuit partition

(c) Sensitivity circuit for calculation

of the variational value

Fig.1 Schematic diagram of our relaxation method

where $m_{1 k}, m_{2 k}$ are integers satisfying

$$
\left|m_{1 k}\right| \leq B, \quad\left|m_{2 k}\right| \leq B
$$

for some sufficiently large $B$.

Assuming that the original circuit in Fig.1(a) has a unique steady-state solution described by $(1)$, then $v_{C}(t)$ and $i_{L}(t)$ satisfies the following determining equation:

$$
\begin{aligned}
& F_{1}\left(v_{C}, i_{L}\right) \equiv i_{C 1}(t)+i_{C 2}(t)=0 \\
& F_{2}\left(v_{C}, i_{L}\right) \equiv v_{L 1}(t)-v_{L 2}(t)=0
\end{aligned}
$$

Now, assume the nonlinear capacitor and inductor are described by

$$
q_{C 2}=\hat{q}_{C 2}\left(v_{C 2}\right), \quad \phi_{L 2}=\hat{\phi}_{L 2}\left(i_{L 2}\right)
$$

Then, we have

$$
i_{C 2}=\frac{\partial \hat{q}_{C 2}}{\partial v_{C 2}} \frac{d v_{C 2}}{d t}, \quad v_{L 2}=\frac{\partial \hat{\phi}_{L 2}}{\partial i_{L 2}} \frac{d i_{L 2}}{d t}
$$

Let us calculate the steady-state response using an iteration technique. Assume the solution at the $j$ th iteration is given by

$$
\begin{aligned}
& v_{C}^{j}(t)=V_{C, 0}^{j}+\sum_{k=1}^{M}\left\{V_{C, 2 k-1}^{j} \cos \nu_{k} t+V_{C, 2 k}^{j} \sin \nu_{k} t\right\} \\
& i_{L}^{j}(t)=I_{L, 0}^{j}+\sum_{k=1}^{M}\left\{I_{L, 2 k-1}^{j} \cos \nu_{k} t+I_{L, 2 k}^{j} \sin \nu_{k} t\right\}
\end{aligned}
$$

To evaluate the solution at the $(j+1)$ th iteration, put

$$
v_{C}^{j+1}(t)=v_{C}^{j}(t)+\Delta v_{C}(t), \quad i_{L}^{j+1}(t)=i_{L}^{j}(t)+\Delta i_{L}(t)
$$

where the variations $\Delta v_{C}(t)$ and $\Delta i_{L}(t)$ are described by $\Delta v_{C}(t)=\Delta V_{C, 0}+\sum_{k=1}^{M}\left\{\Delta V_{C, 2 k-1} \cos \nu_{k} t+\Delta V_{C, 2 k} \sin \nu_{k} t\right\}$

$$
\Delta i_{L}(t)=\Delta I_{L, 0}+\sum_{k=1}^{M}\left\{\Delta I_{L, 2 k-1} \cos \nu_{k} t+\Delta I_{L, 2 k} \sin \nu_{k} t\right\}
$$

Substituting $v_{C}^{j+1}(t), i_{L}^{j+1}(t)$ from (6) into (2), and neglecting the higher-order terms of $\Delta v_{C}(t)$ and $\Delta i_{L}(t)$ in the Taylor expansion of nonlinear terms, we obtain

$$
\begin{gathered}
F_{1}\left(v_{C}^{j}+\Delta v_{C}, i_{L}^{j}+\Delta i_{L}\right)=i_{C 1}\left(v_{C}^{j+1}, i_{L}^{j+1}\right)+i_{C 2}\left(v_{C}^{j+1}\right) \\
\approx i_{C 1}\left(v_{C}^{j}, i_{L}^{j}\right)+\frac{\partial i_{C 1}\left(v_{C}, i_{L}\right)}{\partial v_{C}} \Delta v_{C}+\frac{\partial i_{C 1}\left(v_{C}, i_{L}\right)}{\partial i_{L}} \Delta i_{L} \\
+i_{C 2}\left(v_{C}^{j}\right)+\frac{\partial \hat{q}_{C 2}\left(v_{C}\right)}{\partial v_{C}} \frac{d \Delta v_{C}}{d t}=0 \\
F_{2}\left(v_{C}^{j}+\Delta v_{C}, i_{L}^{j}+\Delta i_{L}\right)=v_{L 1}\left(v_{C}^{j+1}, i_{L}^{j+1}\right)-v_{L 2}\left(i_{L}^{j+1}\right) \\
\approx v_{L 1}\left(v_{C}^{j}, i_{L}^{j}\right)+\frac{\partial v_{L 1}\left(v_{C}, i_{L}\right)}{\partial v_{C}} \Delta v_{C}+\frac{\partial v_{L 1}\left(v_{C}, i_{L}\right)}{\partial i_{L}} \Delta i_{L} \\
-v_{L 2}\left(i_{L}^{j}\right)-\frac{\partial \hat{\phi}_{L 1}\left(i_{L}\right)}{\partial i_{L}} \frac{d \Delta i_{L}}{d t}=0
\end{gathered}
$$

Now, define the residual sources as follows:

$$
\begin{gathered}
\varepsilon_{C}^{j}(t) \equiv i_{C 1}\left(v_{C}^{j}, i_{L}^{j}\right)+i_{C 2}\left(v_{C}^{j}\right) \\
\varepsilon_{L}^{j}(t) \equiv v_{L 1}\left(v_{C}^{j}, i_{L}^{j}\right)-v_{L 2}\left(i_{L}^{j}\right)
\end{gathered}
$$

Since the relation (8) for calculating $\Delta v_{C}$ and $\Delta i_{L}$ is a linear time-varying system, it is not easy to solve even if it is linear. Therefore, we approximate the equations by the time-invariant systems as follows:

$$
\left(\begin{array}{ll}
H_{110} & H_{120} \\
H_{210} & H_{220}
\end{array}\right)=\left(\begin{array}{ll}
\frac{\partial i_{C 1}\left(v_{C}, i_{L}\right)}{\partial v_{C}} & \frac{\partial i_{C 1}\left(v_{C}, i_{L}\right)}{\partial i_{L}} \\
\frac{\partial v_{L_{1}}\left(i_{C}, i_{L}\right)}{\partial v_{C}} & \frac{\partial v_{L} 1\left(v_{C}, i_{L}\right)}{\partial i_{L}}
\end{array}\right)
$$

for $v_{C}=v_{C 0}, i_{L}=i_{L 0}$, and

$$
C_{0}=\left.\frac{\partial \hat{q}_{C 2}}{\partial v_{C 2}}\right|_{v_{C 0}}, \quad L_{0}=\left.\frac{\partial \hat{\phi}_{L 2}}{\partial i_{L 2}}\right|_{i_{L 0}}
$$


where $v_{C 0}$ and $i_{L 0}$ are dc solutions at the operating points. Thus, the relation (8) can be described as follows:

$$
\begin{gathered}
\left(\begin{array}{cc}
H_{110} & H_{120} \\
H_{210} & H_{220}
\end{array}\right)\left(\begin{array}{c}
\Delta v_{C} \\
\Delta i_{L}
\end{array}\right)+\left(\begin{array}{cc}
C_{0} & 0 \\
0 & -L_{0}
\end{array}\right)\left(\begin{array}{c}
\Delta \dot{v}_{C} \\
\Delta \dot{i}_{L}
\end{array}\right) \\
=-\left(\begin{array}{c}
\varepsilon_{C}^{j}(t) \\
\varepsilon_{L}^{j}(t)
\end{array}\right)
\end{gathered}
$$

Thus, we have the equivalent sensitivity circuit shown by Fig.1(c). It can be easily solved by the phasor technique. Observe that, although the convergence ratio may be decreased for the strongly nonlinear circuits, the algorithm is very simple and produces the exact solution after convergence.

The iterations will continue until the variational values $\Delta v_{C}(t)$ and $\Delta i_{L}(t)$ satisfy the following stopping condition:

$$
\begin{array}{r}
\left\|\Delta V_{C}\right\|+\left\|\Delta I_{L}\right\|<\delta \\
\Delta V_{C} \equiv\left[\Delta V_{C, 0}, \ldots, \Delta V_{C, 2 M}\right], \Delta I_{L} \equiv\left[\Delta I_{L, 0}, \ldots, \Delta I_{L, 2 M}\right]
\end{array}
$$

for a sufficiently small $\delta$. Furthermore, if the residual current does not satisfy the following condition:

$$
\left\|\varepsilon^{j}(t)\right\| \equiv \sqrt{\frac{1}{T} \int_{0}^{T}\left(\varepsilon_{C}^{j}(t)\right)^{2}+\left(\varepsilon_{L}^{j}(t)\right)^{2} d t}<\epsilon
$$

for a large $T$ and a small $\epsilon$, then we need to increase the Fourier terms $M$ in (1).

Note that the nonlinear resistive network in Fig.1(a) may have small parasitic capacitors. If they cannot be neglected at the high frequency, we need to take account of the nonlinear capacitors in Fig. 1(a). Thus, the computer efficiency of the algorithm will be decreased according to the number of nonlinear capacitors. In our many examples, we recommend to partition a circuit into subcircuits at only coupling capacitors, whose capacitors voltages are considered as substition voltage sources in Fig.1(b).

\section{SPICE IMPLEMENTATION}

Nowaday, SPICE is widely used for many circuit simulation purposes such as dc-analysis, ac-analysis, transient analysis and so on. Our simulator is implemented by acand dc-analysis of SPICE, and a very simple Fortran program (or C-program).

\section{Implementation algorithm}

0 . A given circuit is partitioned into nonlinear resistive circuits and reactive elements with substitution sources. Considering the amplitudes of the signal and carrier inputs [7], set the highest harmonic $M$ in $(1)$.
Next, choose sufficiently small stopping conditions $\delta$ and $\epsilon$ in (12) and (13), respectively. At first, we draw the dc circuit diagram, and solve it by SPICE $^{2}$. Thus, each capacitor is replaced by a substituting voltage source with a dc-voltage source $V_{C 0}^{0}$, and each inductor by a substituting current source with $I_{L 0}^{0}$.

1. Set $v_{C}^{0}(t)=V_{C 0}^{0}$ and $i_{L}^{0}(t)=I_{L 0}^{0}$. Applying acsweep of SPICE, determine $H_{110}, \ldots, H_{220}, C_{0}$ and $L_{0}$ at zero frequency. Set $j=0$

2. Solve the nonlinear resistive circuit with $(e(t), j(t)$, $\left.v_{C}^{j}(t), i_{L}^{j}(t)\right)$ by dc-analysis of SPICE. In this case, 2-dimensional FFT can be carried out by the application of the one-dimensional FFT to the $\omega_{1}$-components and $\omega_{2}$-components, separately [7]. Thus, we have 2-dimensional Fourier expansions of $i_{C 1}^{j}(t)$ and $v_{L 1}^{j}(t)$.

3. Calculate the responses of nonlinear reactive elements, and describe them by the 2-dimensional Fourier expansions of $i_{C 2}^{j}(t)$ and $v_{L 2}^{j}(t)$. Note that if the reactive elements are linear, we need not apply 2-dimensional FFT.

4. Estimate $\varepsilon_{C}^{j}(t)$ and $\varepsilon_{L}^{j}(t)$ given by (9). Thus, the relation (11) can be easily solved by the phasor technique, and get $\Delta v_{C}(t)$ and $\Delta i_{L}(t)$. If $\left\|\Delta V_{C}^{j}\right\|+\|$ $\Delta I_{L}^{j} \|<\delta$, go to 5 .

Otherwise, set $v_{C}^{j+1}(t)=v_{C}^{j}(t)+\Delta v_{C}$ and $i_{L}^{j+1}(t)=$ $i_{L}^{j}(t)+\Delta i_{L}$, and $j=j+1$. Go to Step 1 .

5. Estimate $\left\|\varepsilon^{j}(t)\right\|$ in (13). If $\left\|\varepsilon^{j}(t)\right\|<\epsilon$, stop. Otherwise, increase $B$ in (1.4) and go to Step 0 .

We have carried out the algorithm with IBM PC loading PSPICE of MicroSim co.

\section{AN ILLUSTRATIVE EXAMPLE}

Consider a mixer circuit shown by Fig.2(a). It has two inputs of

$$
\begin{aligned}
& e_{1}(t)=0.01 \sin 2 \pi \times 50 \times 10^{6} t[V] \\
& e_{2}(t)=0.01 \sin 2 \pi \times 51 \times 10^{6} t[V]
\end{aligned}
$$

We partition the circuit at the two capacitors $C_{1}$ and $C_{2}$, because they are considered as sufficiently large compared

\footnotetext{
${ }^{2}$ Introduce the compensational resistors $R_{c}$ and $-R_{c}$ if the nonlinearity is strong [8].
} 


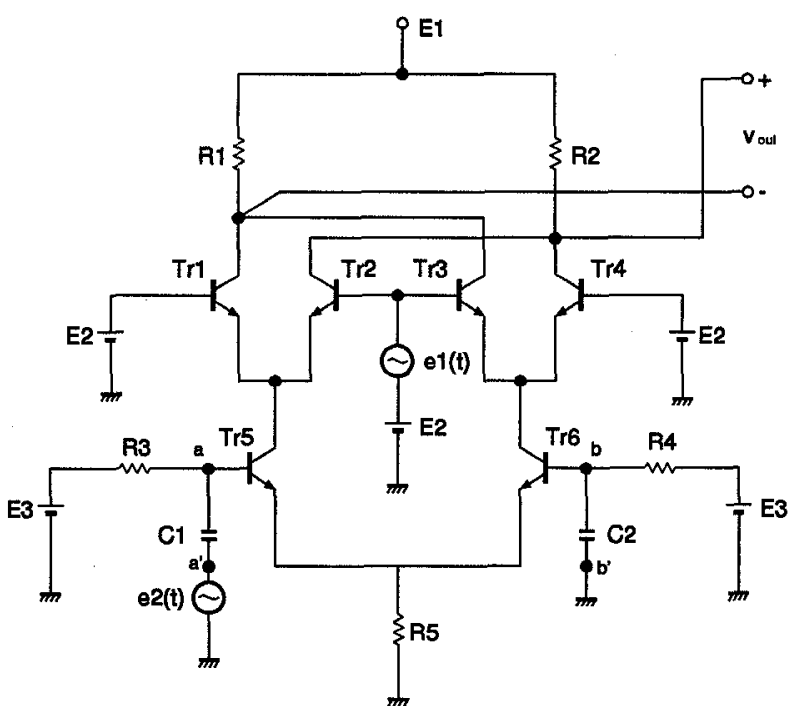

Fig.2(a) Mixer circuit

$R_{1}=R_{2}=100 \Omega, R_{3}=R_{4}=10 k \Omega, C_{1}=C_{2}=0.01 \mu \mathrm{F}$

$R_{5}=200 \Omega, E_{1}=5 \mathrm{~V}, E_{2}=2.5 \mathrm{~V}, E_{3}=12 \mathrm{~V}$

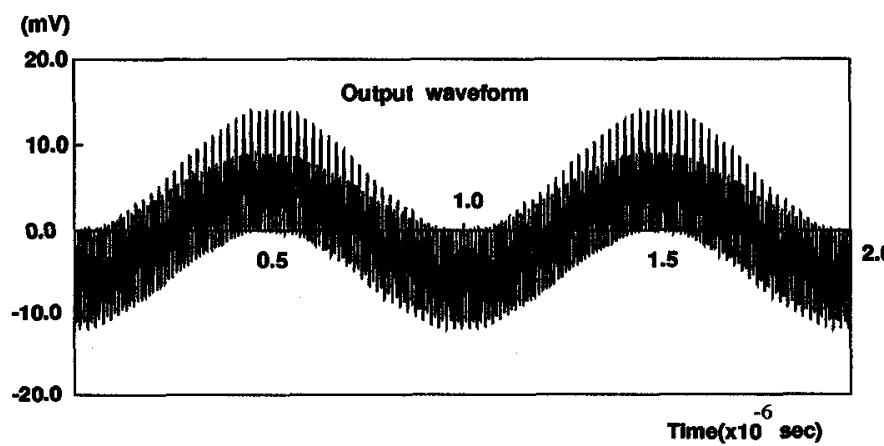

Fig.2(b) Steady-state output waveform

to the parasitic capacitances in the transistors. We assume the waveforms as follows:

$$
\begin{gathered}
v_{i}(t)=V_{i, 0}+\sum_{k=1}^{7} \sum_{n=1}^{7}\left\{V_{i, k n, c 1} \cos \left(k \omega_{1}+n \omega_{2}\right) t\right. \\
+V_{i, k n, c 2} \cos \left(k \omega_{1}-n \omega_{2}\right) t+V_{i, k n, 1 s} \sin \left(k \omega_{1}+i n \omega_{2}\right) t \\
\left.+V_{i, k n, 2 s} \sin \left(k \omega_{1}-n \omega_{2}\right) t\right\}, \quad i=1,2
\end{gathered}
$$

We found that $V_{1 C 0}^{0}=V_{2 C 0}^{0}=2.388[V]$ by the dc-analysis of SPICE. The steady-state response can be obtained in 3 iterations of our algorithm. Note that if we apply a brute force method, it will take an enormous computation time because the relative frequency difference of two inputs is very small, and the response contains a very low frequency of $1 M \mathrm{~Hz}$.

\section{Conclusions and Remarks}

In this paper, we have presented that 2-dimensional Fourier transformation can be efficiently applied to calculate the steady-state response driven by 2 -frequency input signals such as modulators and mixers. The efficiency does not depend on the frequency values. Furthermore, it can be easily modified to the analysis of multiple-frequency inputs greater than two, and to the noise analysis.

We have developed a very simple simulator cosisting of SPICE and a Fortran program, where all of the circuit analyses are implemented by SPICE. Another simple sensitivity analysis and 2-dimensional FFT are carried out by the Fortran program.

Note that, for very high frequency, we need to take into account parasitic capacitors of transistors. If the number is increased, the computer efficiecy will be decreased the computer efficiency. This is a future research problem.

\section{REFERENCES}

[1] K.S.Kundert, G.B.Sorkin and A.Sangjovanni-Vincentelli, "Applying harmonic balance to almost-periodic circuit," IEEE Trans. Microwave Theory Tech., vol.MTT-36, pp.366-378, 1988.

[2] A.Ushida and L.O.Chua, "Frequency-domain analysis of nonlinear circuits driven by multi-tone signals," IEEE Trans. Circuits Syst., vol.CAS-31, pp.766-779, 1984.

[3] M.Okumura, T.Sugawara and H.Tanimoto, "An efficient small signal frequency analysis method of nonlinear circuits with two frequency excitations," IEEE Trans. Computer-aided Design, vol.CAD-9, no.3, pp.225-235, 1990.

[4] M.Okumura, H.Tanimoto, T.Itakura and T.Sugawara, "Numerical noise analysis for nonlinear circuits with a periodic large signal excitation including cyclostationary noise sources," IEEE Trans. Circuits Syst.-I: Fundamental Theory and Applications, vol.CAS-40, no.9, pp.581$590,1993$.

[5] A.Ushida, T.Adachi and L.O.Chua, "Steady-state analysis of nonlinear circuits based on hybrid method," IEEE Trans. Circuits Syst.-I: Fundamental Theory and Applications, vol.CAS-39, no.8, pp.649-661, 1992.

[6] R. Telichevesky and K.Kundert, SpectreRF Primer, Cadence Design System, san Jose, California, July, 1996.

[7] A.Ushida, L.O.Chua and T.Sugawara "A substitution algorithm for solving non-linear circuits with multifrequency components," Int. Jour of Circuit Theory and Applications, vol.15, pp.327-355, 1987.

[8] A.Ushida and L.O.Chua "Steady-state response of nonlinear circuits: A frequency-domain relaxation method, " Int. Jour of Circuit Theory and Applications, vol.17, pp.249-269, 1989. 